\title{
Avoiding a broken heart during anesthesia \\ - Management of Takotsubo Cardiomyopathy
}

\author{
Authors: Daniela Rosinha ${ }^{1}$, Zara Nogueira² ${ }^{2}$ Helena Roxo \\ ${ }^{1}$ Anesthesiology Department, Centro Hospital de Setúbal EPE \\ Anesthesiology Department, Hospital Santa Maria, Centro Hospitalar Lisboa Norte EPE
}

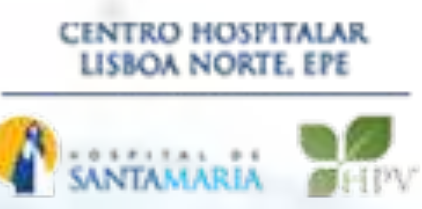

\section{Introduction}

- Takotsubo myocardiopathy (TCM) is a transient cardiac syndrome that involves left ventricular apical akinesis (also known as apical ballooning) and basal hyper contraction that may symptomatically mimic acute coronary syndrome in the absence of coronary disease.

- It's a rare condition usually triggered by a stressful event and patients are often postmenopausal women. According to systematic reviews the recurrence rate of this condition is between $1.5-1.8 \%$, and patients have a higher risk for major cardiac event, stroke or transient ischemic attack.

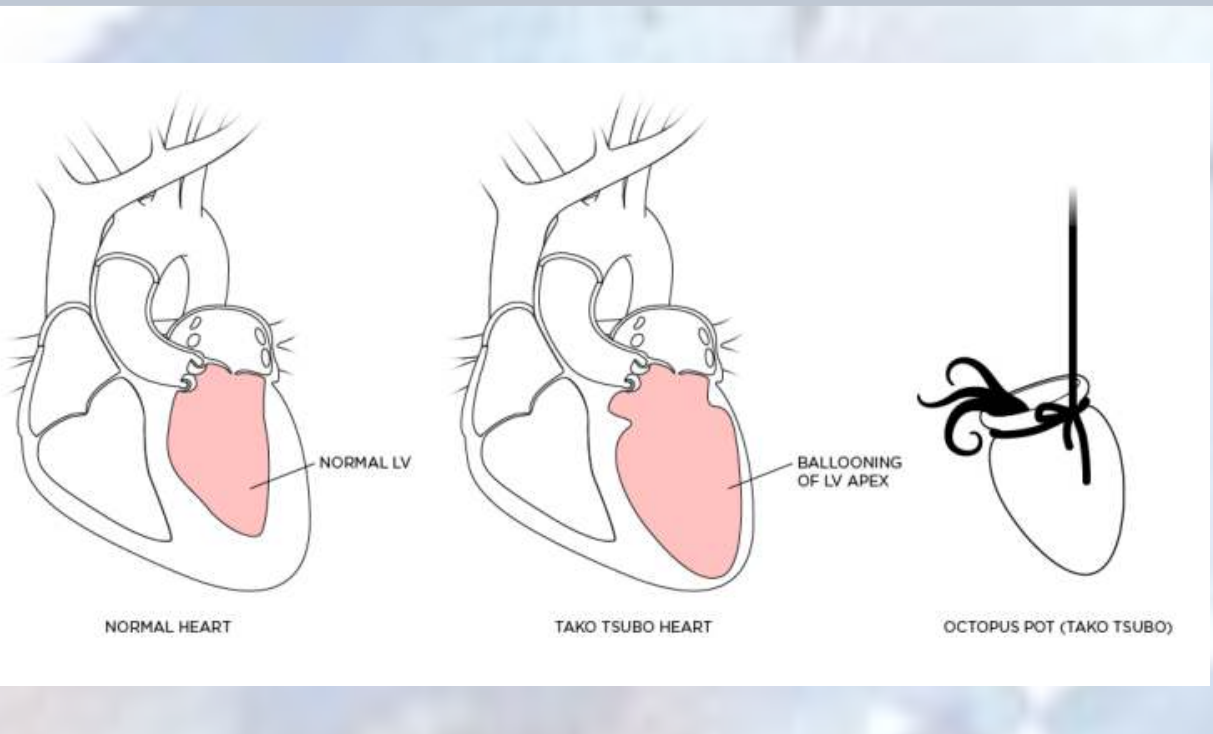

\section{Case Presentation}

- A 75 year-old female patient, ASA III, scheduled for flexible vídeo bronscoscopy (VB) to study a mass in the lower left lobe

- Medical history: hypertension, diabetes mellitus type 2, dislipidemia, hypothyroidism and chronic urticaria.

- Diagnosed with Takotsubo Cardiomyopathy 2 months previously, during omalizumab treatment.

- Complete reversion in 24 hours.

TCM: chest pain + abnormal ECG + decreased systolic function with ejection fraction of $30 \%$ on echocardiography $+\uparrow$ troponin $\mathrm{T}+$ normal coronariography, ventriculography with left ventricular apical akinesis and basal hypertrophy. Complete reversion in $24 \mathrm{~h}$.
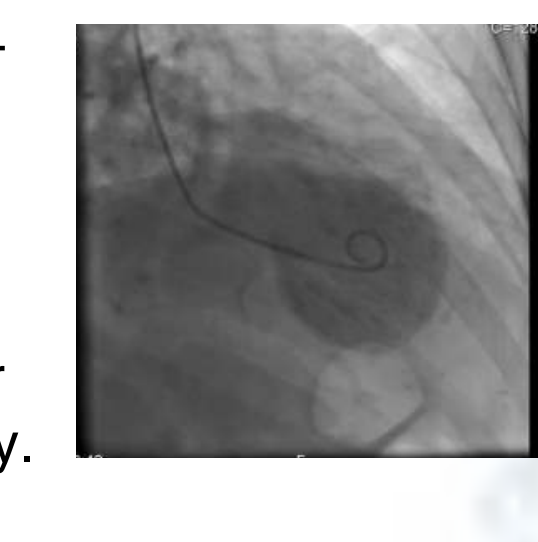

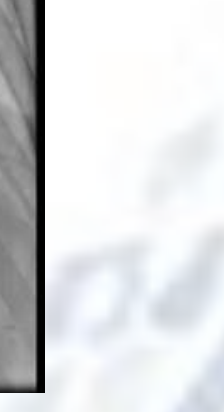
Pre-medication with $2 \mathrm{mg}$ i.v. midazolam, monitored, accompained by nurse, calm environment.
Before entrance to room: 2 mg i.v. midazolam
3 hours in recovery room, monitored, without complications. Uneventful discharge

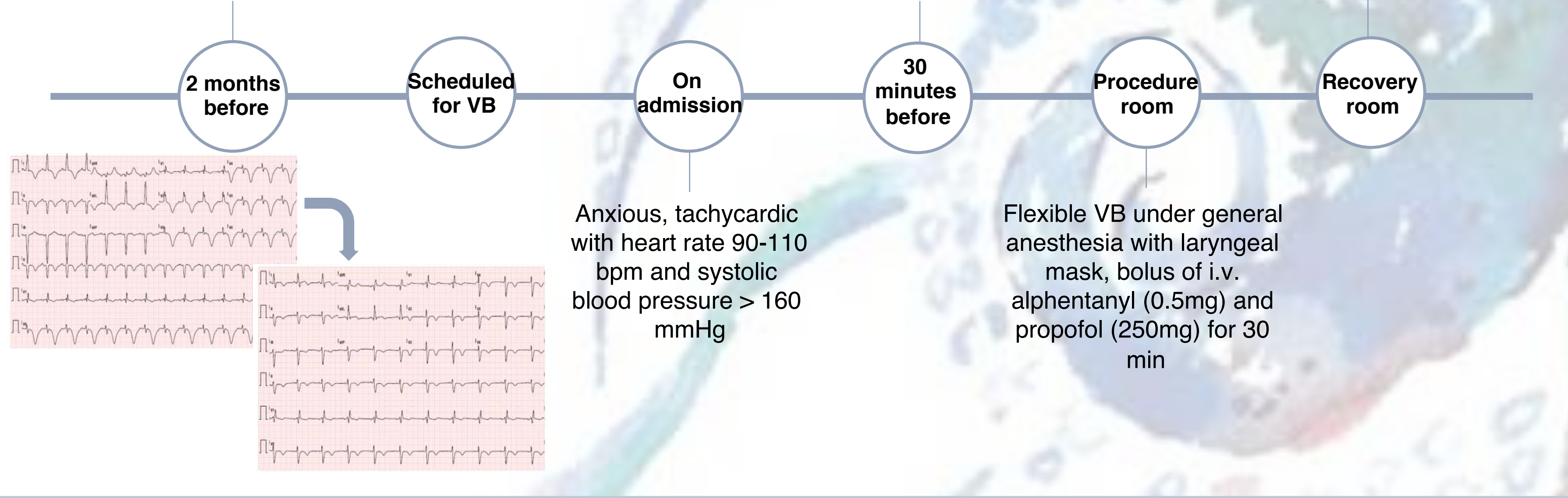

\section{Discussion}

- The avoidance of stressful events and hemodynamic stability seems to have a major role in the perioperative management of these patients, in order to prevent reccurence and major cardiovascular events.

- The manual boluses was the technique chosen in an attempt to prevent any tachycardia/hipertension according to different invasiveness and stimulation of the technique.

- As this was an uneventful procedure and it is a rare disease, this report could help to raise awareness.

\section{Conclusion}

After an exhaustive research we concluded that there's a lack of information about peri and intraoperative management of patients with a previous diagnose of Takotsubo Syndrome. Despite the incidence and recurrence of TCM is believed to be low, anesthesiologists should be aware of this syndrome. 